\title{
Integrating energy efficiency analysis of massive MIMO-based C-RAN
}

\author{
Di Zhang ${ }^{1 *}$ (D), Muhammad Tariq ${ }^{2}$, Shahid Mumtaz ${ }^{3}$, Jonathan Rodriguez ${ }^{3}$ and Takuro Sato ${ }^{1}$
}

\begin{abstract}
Besides the prior study of energy efficiency (EE) that focuses on single component (antenna, radio frequency (RF) chain, circuit, etc.), or limited to one base station (BS) area, in this paper, EE is integrated, investigated with various components (antenna, RF chain, circuit, coverage area) considering in, based on an optimized system model. That is, one optimized multi-BS system model is introduced beforehand with the cloud radio access network (C-RAN) for easier deployment of the selecting/sleeping mechanism. In addition, the system EE is described by an optimization problem while taking more component factors into consideration. While solving, this integrated optimization problem in all time intervals of all BS areas is proved to be equal to an optimization problem of each single BS area within each single time interval. After that, the integrated optimization solution is obtained while adding up the single-BS-singleinterval optimization solutions, whereas the later solution is addressed by an offline decision method plus a convex optimization strategy. Observation from the numerical results demonstrates that the antenna power consumption is the dominant factor of the power consumption, which therefore, the dominant factor of EE performance.
\end{abstract}

Keywords: Energy efficiency, Cloud Radio Access Network, Massive MIMO, 5G, Convex optimization

\section{Introduction}

Energy efficiency (EE) issue receives various attentions in wireless communications nowadays apart from the previous spectrum efficiency (SE) $[1,2]$. In EE study, the energy harvesting $(\mathrm{EH})$ technology is proposed and widely studied recently [3, 4], for the EE optimization [3], simultaneous wireless information and power transfer (SWIPT) [4] and other issues. In addition, the authors of [5] propose one cellular zooming scheme, to divide the cellular coverage area into different ring sections with respect to the distance from the base station (BS) to the user terminal and to select the needed area(s) for zooming in (with users) or out (without users) while cooperating with the cooperative multi-point (CoMP) technology [6]. To step further, a cooperative transmission with BS and antenna selecting/sleeping is investigated in $[7,8]$, and the authors in [9] introduce this selecting/sleeping method into RF chain study. Additionally, in [10], selecting/sleeping mechanism is further investigated in a multi-antenna relay networks. Similarly, in the study of [11], a cellular partition

\footnotetext{
*Correspondence: di_zhang@fuji.waseda.jp

${ }^{1}$ GITS/GITI, Waseda University, Okubo, 3-14-8, 169-0074 Tokyo, Japan

Full list of author information is available at the end of the article
}

zooming scheme is presented to further optimize the cellular zooming scheme of [5]. Compared with [5], the prior study of us in [11] further divides the ring area in [5] into fan sections for zooming in and out, which the system EE performance is progressively boosted up via this method. Although various literature can be found in this regard, yet most of them are limited to one or two components selecting/sleeping cases. Lately, an integrated study has been done in [12] while taking more component power consumptions into consideration for both uplink and downlink analysis. But this work is focused on one BS area scenario.

By contrast, estimation from [13] indicates that while gathering several BSs together for integrated optimization, i.e., machine room sharing, more energy could be saved. Intuitionistic experience also has that while holistically adopting the selecting/sleeping, i.e., taking more components into consideration, EE performance can be further enhanced. Thus while studying EE, an integrated multi-BS multi-component perspective will be better than the previous signal BS or less component ones. One should also note that while taking more elements into
Springer Open

(c) The Author(s). 2016 Open Access This article is distributed under the terms of the Creative Commons Attribution 4.0 International License (http://creativecommons.org/licenses/by/4.0/), which permits unrestricted use, distribution, and reproduction in any medium, provided you give appropriate credit to the original author(s) and the source, provide a link to the Creative Commons license, and indicate if changes were made. 
consideration, although it will be much closer to the reality, but it turns out to be an concave optimization problem while directly solving will cost more resources and time period. In this case, a tradeoff strategy is given in this study.

Additionally, cloud radio access network (C-RAN) provides a platform for the integrated architecture for optimizing $[13,14]$. But due to the one antenna in one remote radio head (RRHs) area configuration, numerous RRHs are needed while deploying. In addition, due to the one antenna RRHs with its small coverage area, zooming scheme and the antenna selecting/sleeping scheme are not considered in prior literature [13].

Therefore, to optimize the system EE, C-RAN is borrowed in this study while introducing massive MIMO antenna arrays to replace the existing LTE antennas in the outdoor environment, and uniformly scheduled several BS resources together to form a "C-RAN." The benefits have been stated in [2]. In addition, other than the previous work that focus on the single component (antenna, radio frequency (RF) chain, etc.) selecting/sleeping scheme, the EE problem within all time intervals is modeled from a comprehensive perspective with more components taking into consideration (machine room, electron circuits, etc.). It is found that the problem is an concave one. To solve it, it is first proved that the global EE optimization problem can be achieved by summing up every optimization solution of each time interval within each single BS area. In addition, the single-time-interval-single-BS-area EE optimization problem can be solved by a linear convex optimization problem plus an offline decision method. Additionally, observation from the results demonstrates that antenna power consumption is the dominant factor of $\mathrm{EE}$ in this system.

The structure of this paper was organized as follows: Section 2 described the proposed system structure. The system model was investigated in detail, including the analysis of its sum rate and energy consumption with regards to the EE definition. In Section 3, the EE problem was modeled into a non-convex preparative optimization problem, and its solution was given. The proposal was verified and compared by the comprehensive computerbased simulation results in Section 4. Finally, the paper was concluded in Section 5.

NOTATIONS: In this paper, the uppercase boldface letters were used to denote the matrix, lowercase boldface letters were the vectors, and normal letters were the scalar quantities, respectively. In addition, $A^{H}$ denoted the Hermitian transposition of a matrix $\boldsymbol{A} ; \boldsymbol{A}^{T}$ denoted the transpose of a matrix $\boldsymbol{A} ; \boldsymbol{A}_{i j}$ was the $(i, j)$ entry of a matrix $\boldsymbol{A}$ with $i$ th raw and $j$ th column; $\|\boldsymbol{A}\|_{F}^{2}$ was the Frobenius 2norm of a matrix $\boldsymbol{A} ; \mathbb{E}$ was the expectation operation; and $\stackrel{\text { a.s. }}{\longrightarrow}$ denoted the almost sure convergence, respectively.

\section{Proposed system model and analysis of the transmission rate and energy consumption}

As the definition of EE $[15,16]$, in order to investigate the $E E$, achievable sum rate and consumed energy expression should be achieved beforehand. Thus in the following subsections, the optimized system model, the sum rate, and its consumed energy investigation based on this model were addressed beforehand.

\subsection{The proposed system model}

Typically, C-RAN consists of baseband unit (BBU), RRHs, and high bandwidth low latency connections between the RRHs and BBU [13]. The benefits of C-RAN reside in its joint information processing and resource scheduling, which can be executed via the virtual base station (VBS) that connects with a general purpose platform (GPP) server.

Consequently, an example of the proposed system model can be shown in Fig. 1. In addition, while uniformly scheduling several BSs' resources, the "BBU" sections are separated into two parts: plant and control center, switcher connected to the core network. Furthermore, unlike several BBUs within one C-RAN cluster, here the system is further modified by connecting all of its connecting BSs to one "BBU" for uniformly resource scheduling purpose. The benefit of this strategy is stated by [2]. Here, the latency issue is left for further study while adopting the CoMP-JP with cooperating cellular in line with the majority work of this, such as [17].

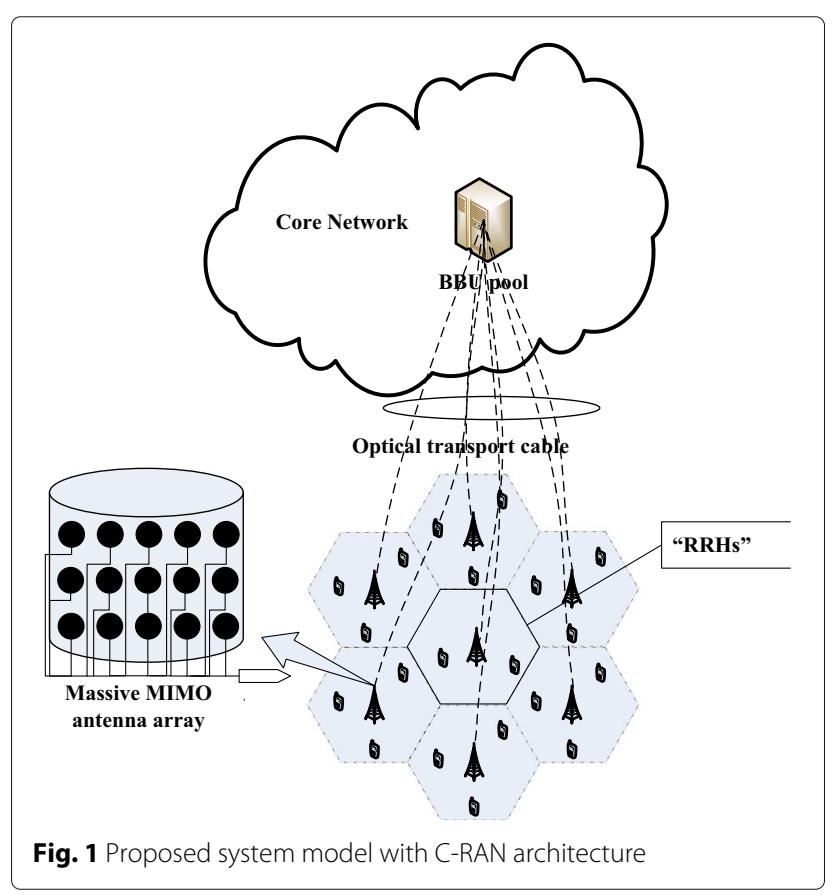




\subsection{The transmission rate analysis}

Take the hypothesis that there are $K$ active UEs that each of them associating with $b$ active antennas for its transmission. Suppose that all of the UE's requests are routed back to the uniform "BBU" through backhaul links with CoMP-JP. Moreover, power of each antenna is equally assumed to be $P_{a}$. By ignoring the inter-cluster interferences from the neighboring $\mathrm{BS}$ area $[2,11]$, further take $\mathbf{h}_{k}$, a $1 \times M$ complex-Gaussian vector with zeromean-complex-Gaussian entries, and variance $\frac{1}{2}$ for each dimension, as the channel from BS to UE $k$ [18]. While assuming component carrier (CC) bandwidth assigned to each UE is $B_{\mathrm{CCs}}$ where CC is sufficient enough for $K$ UEs. In line with study in $[2,11,18]$, with $K, M$ growing large with a constant ratio $M / K$, by an equal power allocation, achievable rate of UE $k$ can be given by [2]

$$
\begin{aligned}
R_{k} & =\mathbb{E}\left\{B_{\mathrm{CCs}} \log _{2}\left(1+\frac{\rho}{\operatorname{tr}\left[\left(\boldsymbol{H H}^{H}\right)^{-1}\right]}\right)\right\} \\
& \geq B_{\mathrm{CCs}} \log _{2}\left(1+\frac{\rho}{\mathbb{E}\left\{\operatorname{tr}\left[\left(\boldsymbol{H} \boldsymbol{H}^{H}\right)^{-1}\right]\right\}}\right) \\
& \stackrel{\text { a.s. }}{\longrightarrow} B_{\mathrm{CCs}} \log _{2}\left(1+\rho \frac{M-K}{K}\right),
\end{aligned}
$$

where $\rho=P_{k} / P_{n_{k}}$, with $P_{n_{k}}$ the noise power for each user $k$. Here the second step is obtained by employing the Jensen's inequality, whereas the approximate equation by the third step is obtained in line with prior studies of $[2,10]$. This is the "channel hardening" effect in massive MIMO, which means that with $K, M$ growing large, the off-diagonal $\mathbf{H}^{H} \mathbf{H}$ effects become increasing weaker compared with the diagonal matrix of $\mathbf{H}[19,20]$. The sum rate within each C-RAN under this assumption turns out to be the total transmission rate of the overall BS areas. In addition, for the sake of compactness, it is further assumed that the UE number is equal among different cellular areas. Thus, the sum rate, while denoting $B$ as the total cellular number of this system, turns out to be

$$
R_{\text {sum }}=\sum_{i=1}^{B} \sum_{j=1}^{K} R_{i, j}^{k},
$$

where $R_{i, j}^{k}$ is equal to $R_{k}$. With this sum rate in hand, the left work is the power consumption analysis, which was addressed by the following sections.

\subsection{The energy consumption analysis}

Energy consumption of C-RAN, typically, can be summarized into the dynamic energy consumption plus the constant energy consumption parts. The dynamic energy consumption mainly comes from the transmission (for instance, antenna, RF chain, and circuit to serve the transmission) and coverage area maintaining [2]. In contrast, the constant energy consumption mainly results from the machine room and other equipments, which seldom light is casted on in prior studies. Under this circumstance, total energy consumption follows [21]

$$
P_{\text {clu }}(t)=\sum_{i=1}^{B}\left(p_{i}^{\mathrm{dyn}, \mathrm{BS}}+p_{i}^{\mathrm{fix}, \mathrm{BS}}\right),
$$

where $p_{b}^{\mathrm{dyn}, \mathrm{BS}}, p_{b}^{\mathrm{fix}, \mathrm{BS}}$ yield the dynamic energy consumption of each BS area (to serve the UEs and maintain the BS coverage area) and constant energy consumption of each BS area. Additionally, the dynamic energy consumption can be further elaborated by

$$
p_{b}^{\mathrm{dyn}, \mathrm{BS}}=\sum_{j=1}^{K}\left(\frac{1}{\eta} P_{j}^{k}+P_{j}^{c}+P_{j}^{\mathrm{RF}}+P_{j}^{\mathrm{cc}}\right) .
$$

Here, $P_{j}^{k}$ is the transmission rate energy consumption (equal to $P_{k}$, the allocated antenna energy power of UE $k$ with rate $R_{i, j}^{k}$ ). In addition, $P_{j}^{\mathrm{RF}}, P_{j}^{c}, P_{j}^{\mathrm{cc}}$ are the RF chain, circuit, and the allocated coverage area maintaining energy consumption, respectively. Furthermore, $\eta$ denotes the power amplifier (PA) efficiency with value $0<\eta<1$.

As discussed before, the goal of this paper is to explore the EE merits of this proposal while taking more components into consideration. In order to describe this comprehensive EE with mathematics model, two lemmas are given beforehand.

Lemma. (lemma 3.1 in [22]) only consider the downlink transmission while setting the power of uplink transmission period to be zero ${ }^{1}$, the optimal power allocation for an epoch $t$ is $P_{j}^{k}>0$ for power on time period $\tau^{o n} \subseteq$ $(t(i-1), t(i)]$ (downlink), and $P_{j}^{k}=0$ for the remaining time periods $\tau^{\text {off }} \subseteq(t(i-1), t(i)]$ (uplink). The following identities also hold: $\tau^{\text {on }} \cap \tau^{\text {off }}=\phi, \tau^{\text {on }} \cup \tau^{\text {off }}=$ $(t(i-1), t(i)]$.

\section{Proof. See Lemma 3.1 in [22].}

Lemma. By assuming the arrive of UE request obeys a Poisson point process (PPP) distribution with mean $\mu$ variance $\sigma^{2}$ of all epoch time, and $T=\sum_{t=1}^{T}\left(t_{i-1}, t_{i}\right]$. Optimal power allocation can be obtained while adding the optimal power allocation in all time intervals, and the performance of UEs obeys a PPP distribution with mean $\mu$, variance $\sigma^{2}$. In this case, UEs' performance within the total time intervals obeys a PPP distribution with mean $T \mu$, variance $T \sigma^{2}$.

Proof. See Appendix. 


\section{The energy efficiency model and its solution}

The EE optimization model and its solution of this study are addressed in this section. As discussed before, EE is defined as the achievable sum rate divided by the consumed energy. Hence, in line with prior study [20], the global EE optimization problem of this modified system (within all time intervals of all BS areas), while integrated taking the antenna, RF chain, circuit, and dynamic and constant power into consideration, can be described as

$$
\max _{P_{i, j}^{k}, P_{i, j}^{\mathrm{RF}}} \int_{0}^{T} \sum_{i=1}^{B} \sum_{j=1}^{K} \frac{R_{i, j}^{k}}{P_{\mathrm{clu}}} d t
$$

subject to:

$$
\begin{aligned}
& C 1: \int_{0}^{T} \sum_{i=1}^{B} \sum_{j=1}^{K}\left(\frac{1}{\eta} P_{i, j}^{k}+P_{i, j}^{c}+P_{i, j}^{\mathrm{RF}}\right) d t \leq P_{T}^{\mathrm{total}}, \\
& C 2: \int_{0}^{T} \sum_{i=1}^{B} P_{b}^{\mathrm{dyn}, \mathrm{BS}} d t \leq P_{\mathrm{bs}}^{\mathrm{total}}, \\
& C 3: \int_{0}^{T} \sum_{i=1}^{B} \sum_{j=1}^{K}\left(\left[\frac{R_{i, j}^{k}}{\log _{2}\left(1+\rho \frac{M-K}{K}\right)}\right]\right) d t \leq N_{\mathrm{CCs}}^{\mathrm{total}}, \\
& C 4: \int_{0}^{T} \sum_{i=1}^{B} \sum_{j=1}^{K} N_{i, j}^{a} d t \leq N_{\mathrm{bs}}^{a, \text { total }}, \\
& C 5: \int_{0}^{T} \sum_{i=1}^{B} \sum_{j=1}^{K} N_{i, j}^{\mathrm{UE}} d t \leq N_{b s}^{r f, \text { total }}, \\
& C 6: P_{i, j}^{k}, P_{i, j}^{c}, P_{i, j}^{\mathrm{RF}}, P_{b}^{\mathrm{dyn}, \mathrm{BS}} \geq 0, \forall k,
\end{aligned}
$$

where $P_{i, j}^{k}, P_{i, j}^{c}, P_{i, j}^{\mathrm{RF}}$ are of the same value as $P_{j}^{k}, P_{j}^{c}, P_{j}^{\mathrm{RF}}$. Here $P_{T}^{\text {total }}, P_{b s}^{\text {total }}, N_{C C s}^{\text {total }}, N_{b s}^{a, \text { total }}, N_{b s}^{r f \text {,total }}$ yield the total transmission power, total BS power, the number of $\mathrm{CC}$, the number of antenna, and the number of RF chain, respectively. Further, $N_{i, j}^{a}$, $N_{i, j}^{\mathrm{UE}}, N_{\mathrm{bs}}^{\mathrm{rf} \text { total }}$ denote the number of antenna allocated to each UE, number of UE indicator, and the total number of RF chain within one cluster, respectively. Note that here $\mathrm{C} 1, \mathrm{C} 2$ specify that in one cluster, the total transmission power should be no greater than the allocated power, and the total cellular power consumption should be no greater than the total $\mathrm{BS}$ power, respectively. In addition, C3, C4, C6 denote that in one cluster, the number constraints of $\mathrm{CC}$, antenna, as well as the power constraints, should be satisfied.
Whereas C5 yields the UE number cannot exceed the RF chain number. This is because of the assumption that one active RF chain could only serve one active UE, in line with the prior MIMO structure, for the sake of compactness.

The EE optimization problem, although, has been addressed so far and can be solved by the concave optimization method, but it will cost more resources and calculation time due to the constraint C3 comparing with the linear searching method. Thus, another purpose of this paper is to find an alternative method to simplify the processing. Fortunately, in line with prior study [21], it is noted that if $C_{k}$ was taken as the used CCs of the previous UEs, a minimum CC selection method can be obtained beforehand, whereas $I_{m}(t)$ is defined as

$$
I_{m}(t)=\left\{\begin{array}{l}
1, C_{k} \in \Omega_{m}, C_{k}=\arg \min _{C_{k} \in \Omega_{m}} P_{j}^{k} \\
0, \text { otherwise, }
\end{array}\right.
$$

with $C_{k}, \Omega_{m}$ denote the optimal CC for $k$ th UE and the set of CCs. For the left optimization problem, we dig more and find the following Corollary existing.

Corollary. According to Lemma 2, the binary value of power allocation $P_{i, j}^{k}$ and its time distribution $\tau^{o n}$ both obey the identically Poisson distribution in every time interval $(t(i-1), t(i)]$. While linearly adding every individual power allocation within each time interval, it turns out to be the optimal solution of the total time intervals. This can be proved as follows.

Proof. In $\tau^{\text {on }}$ time period, whenever there is a transmission request, power allocation is triggered with a constant value of $P_{i, j}^{k}$ (proof of Lemma 1 in [22]). Otherwise, in $\tau^{\text {off }}$ period, no power is allocated. This is because $\tau^{\text {on }}$ and $P_{i, j}^{k}$ display the same performances like the active UEs' existences with their on-off binary values. Thus, it is clear that the binary values of $\tau, P_{i, j}^{k}$ are of the same distribution performances just like UEs.

With the aforementioned Lemma 1, Lemma 2, and Corollary 1 in hand, the global EE optimization solution can be obtained while summing up the single time interval EE optimization problems other than the aforementioned integration to the total time intervals. In addition, the single time interval EE optimization problem is the same among different time periods on condition that among each of them, the UEs are performing the same distribution. Thus once the optimal solution is obtained in a time interval, the global optimization is achieved. In 
this case, while removing the integration to the total time intervals, the problem can be further described as follows

$$
\max _{P_{i, j}^{k}, P_{i, j}^{\mathrm{RF}}} \sum_{i=1}^{B} \sum_{j=1}^{K} \frac{R_{i, j}^{k}}{P_{\mathrm{clu}}},
$$

subject to

$$
\begin{aligned}
& C 1: \sum_{i=1}^{B} \sum_{j=1}^{K}\left(\frac{1}{\eta} P_{i, j}^{k}+P_{i, j}^{c}+P_{i, j}^{\mathrm{RF}}\right) \leq P_{T}^{\mathrm{total}}, \\
& C 2: \sum_{i=1}^{B} P_{b}^{\mathrm{dyn}, \mathrm{BS}} \leq P_{\mathrm{bs}}^{\mathrm{total}}, \\
& C 3: \sum_{i=1}^{B} \sum_{j=1}^{K}\left(\left\lceil\frac{R_{i, j}^{k}}{\log _{2}\left(1+\rho \frac{M-K}{K}\right)}\right]\right) \leq N_{\mathrm{CCs}}^{\mathrm{total}}, \\
& C 4: \sum_{i=1}^{B} \sum_{j=1}^{K} N_{i, j}^{a} \leq N_{\mathrm{bs}}^{a, \mathrm{total}}, \\
& C 5: \sum_{i=1}^{B} \sum_{j=1}^{K} N_{i, j}^{\mathrm{UE}} \leq N_{\mathrm{bs}}^{r f, \mathrm{total}}, \\
& \text { C6 }: P_{i, j}^{k}, P_{i, j}^{c}, P_{i, j}^{\mathrm{RF}}, P_{b}^{\mathrm{dyn}, \mathrm{BS}} \geq 0, \forall k .
\end{aligned}
$$

Note that the purpose of $\mathrm{C} 3$ is to find the optimal CC solution under $\mathrm{CC}$ number constraint, which has less relationship with the power allocation. In this case, it can be solved via an offline method. That is, whenever request coming, select the least $\mathrm{CC}$ number for its transmission under $\mathrm{CC}$ constraint in (7). The remaining part after this selection turns out to be a component selection and power allocation problem with respect to the achievable transmission rate. In addition, without loss of generality, it is further assumed that each antenna consumes the same power once activated, as well as the circuit, RF chain.

In addition, similar to Lemma 2, while adding up all users' location performances within every BS areas, it is the UEs' location performances of the whole cluster. Moreover, the PPP distribution of UE within one cluster, with large time range, can be taken as a sum of separate PPP distribution of each BS area. Whereas in each area, the UE displays the same PPP distribution but with a smaller range. Thus, the optimal solution can be obtained by optimizing the EE problem of one BS area within one time interval. Which is, the remaining problem can be decomposed as a optimization problem with

$$
\max _{P_{j}^{k}, P_{j}^{\mathrm{RF}}} \sum_{k=1}^{K} \frac{R_{i, j}^{k}}{P_{j}^{k}+P_{j}^{\mathrm{RF}}},
$$

subject to

$$
\begin{aligned}
& C 1: \sum_{j=1}^{K}\left(\frac{1}{\eta} P_{j}^{k}+P_{j}^{c}+P_{j}^{\mathrm{RF}}\right) \leq P_{T}, \\
& C 2: \sum_{j=1}^{K} N_{j}^{a} \leq N_{\mathrm{bs}}^{a}, \forall b, \\
& C 3: \sum_{j=1}^{K} N_{j}^{\mathrm{UE}} \leq N_{\mathrm{bs}}^{\mathrm{rf}}, \forall t, \\
& C 4: P_{j}^{k}, P_{j}^{c}, P_{j}^{\mathrm{RF}} \geq 0, \forall k,
\end{aligned}
$$

whereas the global optimization solution is the summarization of each of this single-time-interval-single-BS-area optimization problem. To this optimization problem, it is proved by Theorem 1 in [23] that, if we define a maximum weighted solution with $S^{*}=\max \left(P_{j}^{k}, P_{j}^{R F}\right)=$ $\sum_{k=1}^{K} R_{j}^{k *} /\left(P_{J}^{k}+P_{j}^{R F}\right)^{*}$, and further suppose the problem has an optimal solution $S^{*}$. Optimal solution of the EE problem in (10), if any, should satisfy the following constraint

$$
\begin{aligned}
& \max \left(P_{j}^{k}, P_{j}^{\mathrm{RF}}\right)-S^{*}\left(P_{j}^{k}+P_{j}^{\mathrm{RF}}\right)^{*} \\
& =\sum_{k=1}^{K} R_{j}^{k *}-S^{*}\left(P_{j}^{k}+P_{j}^{\mathrm{RF}}\right)^{*}=0,
\end{aligned}
$$

and its optimal solution with $\max \left(P_{j}^{k}, P_{j}^{\mathrm{RF}}\right)$ must be equal to $S^{*}$ if any. Note that RF chain power consumption has nothing to do with the achievable sum rate although it is needed for the transmission. Under this circumstance, while searching for the optimal solution, it will converge to zero. To avoid this, in line with [24], we assume that in each search step, the number of RF chain is equal to the number of antenna in order to satisfy the transmission requirement.

After this, the extreme point existence is verified by the Lagrange method as shown in (13) and (14). As we can see, it always has an extreme point on condition that (13) is equal to zero and (14) lower than zero. Thus, at least one optimal solution of our EE optimization problem exists. In addition, this optimal solution can be straightforward obtained while comparing all of those extreme points and keeping the one with best EE performance.

$$
\begin{aligned}
& \frac{\partial \max \left(P_{j}^{k}, P_{j}^{\mathrm{RF}}\right)}{\partial P_{j}^{k}}=\frac{\partial \sum_{j=1}^{K} \frac{R_{j}}{P_{j}^{k}+P_{j}^{\mathrm{RF}}}}{\partial P_{j}^{k}} \\
& =\sum_{j=1}^{K} \frac{B_{\mathrm{CCs}}\left(\frac{M-K}{\ln 2\left[K N_{0}+P_{j}^{k}(M-K)\right]}-\log _{2} \frac{K N_{0}+P_{j}^{k}(M-K)}{K N_{0}}\right)}{\left(P_{j}^{k}+P_{j}^{\mathrm{RF}}\right)^{2}},
\end{aligned}
$$




$$
\begin{aligned}
& \frac{\partial^{2} \max \left(P_{j}^{k}, P_{j}^{\mathrm{RF}}\right)}{\partial\left(P_{j}^{k}\right)^{2}}=\frac{\partial^{2} \sum_{j=1}^{K} \frac{R_{j}^{k}}{P_{j}^{k}+P_{j}^{\mathrm{RF}}}}{\partial\left(P_{j}^{k}\right)^{2}}= \\
& -\sum_{j=1}^{K} \frac{B_{\mathrm{CCs}}\left(\frac{(M-K)^{2}}{\ln 2\left[K N_{0}+P_{j}^{k}(M-K)\right]^{2}}+\frac{M-K}{\ln 2\left[K N_{0}+P_{j}^{k}(M-K)\right]}\right)}{\left(P_{j}^{k}+P_{j}^{\mathrm{RF}}\right)^{2}} \\
& -\sum_{j=1}^{K} \frac{2 B_{\mathrm{CCs}}\left(\frac{M-K}{\ln 2\left[K N_{0}+P_{j}^{k}(M-K)\right]}-\log _{2} \frac{K N_{0}+P_{j}^{k}(M-K)}{K N_{0}}\right)}{\left(P_{j}^{k}+P_{j}^{\mathrm{RF}}\right)^{3}} .
\end{aligned}
$$

As all constraints are in linear functions now, one can use the linear searching method to obtain the optimal solution of (10) with constraints (11) plus an offline searching method of $\mathrm{CC}$. That is, in each step, search the optimal solution with every possible combination of the constraints, compare them, and only keep the best one. As the linear constraints, one can obtain the optimal solution within a much shorter time. Whereas the searching can be achieved by IBM Cplex (refer to IBM ILOG CPLEX Optimization Studio V12.60 documents) or MATLAB-based linear programming (LP) [25].

\section{Numerical analysis and results}

Here in the numerical analyses and simulation setups of this paper, the simulation parameters in line with $[9,26]$ are employed, which is shown in Table 1. In addition, in each BS area, it is assumed that the active UE number is 20 in one BS area. In this case, achievable sum rate of each cellular within one time interval is the active UE's sum rate. In addition, the sum rate of the system cluster is the amount rate of each BS area within the total time intervals. Another, parts of the UE's location distribution performance is shown in Fig. 2. Note that due to the limit space and tedious generation, here we only gave one figure to show this distribution as an example of our

Table 1 Simulation components

\begin{tabular}{ll}
\hline Parameters & Value \\
\hline Antenna number per RRH & 100 \\
RRH number & 10 \\
Machine room & $480 \mathrm{~W}$ \\
Number of UE per BS area & 20 \\
Mean transmission rate per UE & $200 \mathrm{MHz}$ \\
Circuit power of per RF chain $P_{j}^{\mathrm{RF}}$ & $160 \mathrm{~mW}$ \\
Constant circuit power $P_{j}^{c}$ & $160.8 \mathrm{~W}$ \\
PA efficiency $\eta$ & $35 \%$ \\
Power of antenna $P_{j}^{k}$ & $37 \mathrm{dBm}$ \\
\hline
\end{tabular}

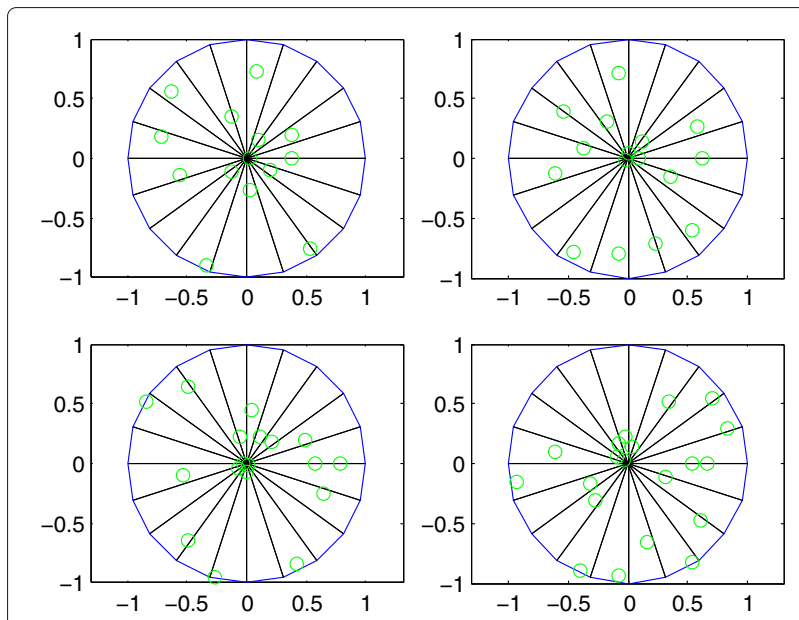

Fig. 2 Location distribution of active UE in one cellular coverage area

simulation, whereas the number of active UEs is growing from 14 to 20 . As shown in this figure, the green bubble yields the location of one active UE whereas the blue circle is the coverage area edge. The $x$ and $y$ coordinate axes denote the normalized range of the cellular area. Note that for easy discrimination, here one cell coverage circle area is divided into 20 parts for better display of the active UE's position in this simulation. The reason to adopt the PPP distribution is that the location of active UEs randomly disperses in cluster area, which is similar to the UE performance in the real world.

Additionally, we further randomly generate the transmission rate. In the simulation here, we evaluate the performance of component selection and EE performance in one cellular area. The randomly generated transmission rate can be found in Table 2 with its simulation results in Figs. 3 and 4. Here, Fig. 3 yields the number of selected components and Fig. 4 is the EE performance. As the PPP distribution of the UE's position within each cell and randomly generated transmission rate, the curve of EE performance in Fig. 4 goes up and down. That is because in each step, the randomly generated transmission rate results in the up and down curves of the energy consumption, which in turn, the up and down curves of EE performance.

One can figure out from Fig. 3 that at first, the number of selected components decreases while CC increasing. Afterwards, CC has less effect to the component selection performance while arriving at the optimal one; $C_{k}$ here in this simulation. Thus on the condition that each pair of components consumes an equal power, $C_{k}$ will be the one with the least component number and $\mathrm{CC}$ value, $35 \mathrm{MHz}$ here. This can be further confirmed in Fig. 4 , where $B_{\mathrm{CCs}}$ with 35 and $40 \mathrm{MHz}$ displays the same EE performances. Thus, it can be concluded that before $C_{k}$, the number of selected components decreases and EE performance 
Table 2 Value of transmission rate request

\begin{tabular}{lrlr}
\hline Number of UE & Value $(\mathrm{M} / \mathrm{s})$ & Number of UE & Value $(\mathrm{M} / \mathrm{s})$ \\
\hline 1 & 64.873 & 11 & 180.217 \\
2 & 317.714 & 12 & 33.529 \\
3 & 124.486 & 13 & 91.591 \\
4 & 211.413 & 14 & 365.335 \\
5 & 66.260 & 15 & 60.951 \\
6 & 240.793 & 16 & 330.327 \\
7 & 105.189 & 17 & 215.337 \\
8 & 261.632 & 18 & 398.454 \\
9 & 275.686 & 19 & 31.270 \\
10 & 299.261 & 20 & 177.071 \\
\hline
\end{tabular}

increases while $B_{\mathrm{CCs}}$ increases. Yet when $B_{\mathrm{CCs}}$ arrived at $C_{k}$, simply increasing the $C C$ has little effect to the EE performance. The best $B_{C C s}$ is $C_{k}$ and the optimal solution of EE optimization problem is obtained while solving (10), by taking $C_{k}$ with the value of $35 \mathrm{MHz}$.

By assuming one cluster consisting of 10 massive MIMO BSs, Figs. 5 and 6 are the component selection and EE performance of the optimized system model within one cluster. As shown, similar to Figs. 3 and 4, because of the random transmission rates, EE curves here go up and down. One can further confirm from these two figures that the optimal $B_{\mathrm{CCs}}$ is $35 \mathrm{MHz}$.

To further verify the validity of our proposal, EE performance of this proposal and the prior state-of-the-art proposal in [7] with BS and antenna selecting and switching off scheme are further compared. The result can be found in Fig. 7. It is clear that our proposal can enhance the EE performance while taking more elements into consideration. That is, by the integrated proposal, one can

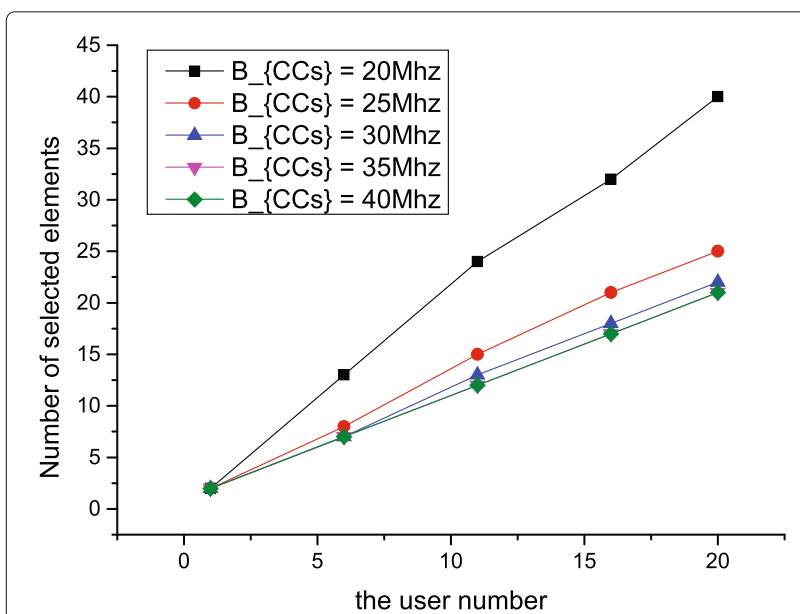

Fig. 3 Component selection performance of our scheme, one cellular area

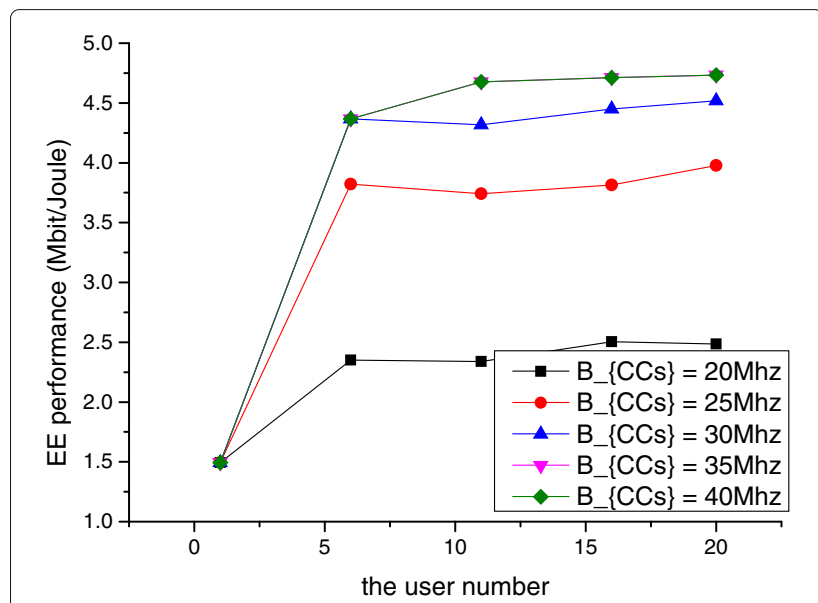

Fig. 4 Energy efficiency performance of our scheme, one cellular area

select the optimal number of components for the transmission while switching off the unwanted ones. But in [7], the unwanted power consumptions of other components (for instance, RF chain, circuit) are still needed for the transmission. One can see from Fig. 7 that, although the EE performance is enhanced by our proposal, the other components (RF chain, electrons, etc.) have less effect to the EE performance comparing with the antennas because of the close gap between the two. Thus, the antenna power consumption is the dominating factor of the power consumptions, which in turn, the dominating factor of system EE performance.

\section{Conclusions}

In this paper, an optimized system model is proposed for economical deployment in outdoor environment and better adoption of the selecting/sleeping mechanism. Afterwards, the system EE performance is modeled into a

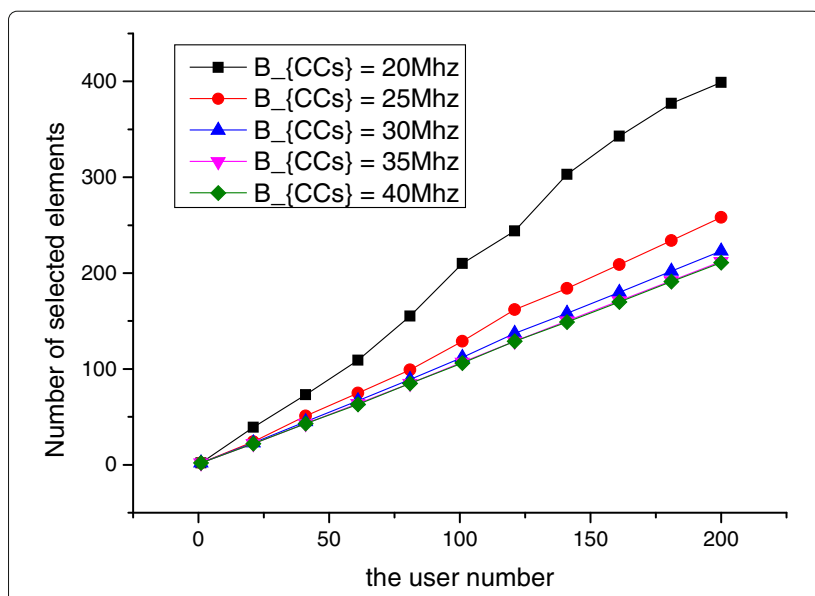

Fig. 5 Component selection performance of our proposal in one cluster 


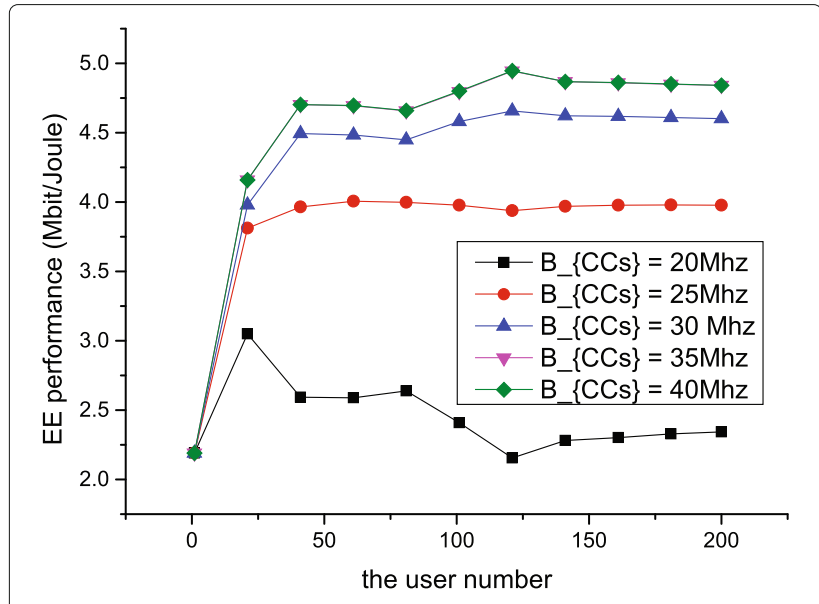

Fig. 6 Energy efficiency performance of our proposal in one cluster

global optimization problem. Solution of this problem is obtained while summing up the solution of each BS coverage area within a single-time-interval-single-BS-area optimization problem. In addition, the single-timeinterval-single-BS-area optimization problem is solved by a linear convex optimization problem plus an offline selection method. Numerical results manifest that the proposal here displays better EE performance comparing with the previous proposal, by further reducing the reminder component power consumptions other than the antenna, RF chain, or other component alone. In addition, $\mathrm{CC}$ has great effect to the performance before reaching the optimal one and little influence after this. The numerical results further indicate that among the component power consumptions, antenna power consumption is the dominant one.

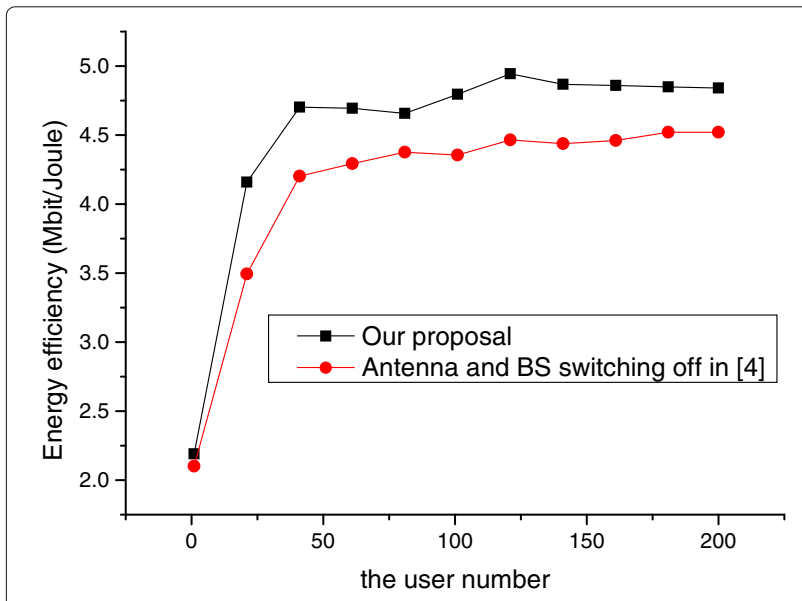

Fig. 7 Energy efficiency comparison between our proposal and proposal in [7]

\section{Endnote}

${ }^{1}$ In this paper, we try to solve the EE optimization problem in downlink, the uplink EE optimization although not considered here, but can be also obtained while setting the downlink power as zero, as discussed in the following sections.

\section{Appendix}

It is difficult to prove the lemma directly, but the proof could be done by some division and summarization methods, i.e., to prove that the sum distribution $\sum_{t=1}^{T} \mathrm{UE}_{t}$ of independent identically distributed Poisson distribution of each time period $\mathrm{UE}_{t}$ was a Poisson distribution. Whereas this can be confirmed by some linear operations. Which is, according to the basic property of expectation value and variance in statistics, expectation calculation of the independent identically distributed Poisson distribution is a linear operation, and the variance is independent. Thus, it has

$$
\begin{gathered}
E(\mathrm{UE})=\sum_{t=1}^{T} E\left(\mathrm{UE}_{t}\right)=T \mu, \\
\operatorname{var}(\mathrm{UE})=\sum_{t=1}^{T} \operatorname{var}\left(\mathrm{UE}_{t}\right)=T \sigma^{2} .
\end{gathered}
$$

In addition, according to the deduction of Lemma 1, in each time period, the optimal power, if any, should be allocated with a constant value. Suppose this constant value is $P_{k}$, where $P_{k}>0, t \in(t(i-1), t(i)]$. By assuming "EE" as the optimal solution operator, according to Jensen's inequality, it follows

$$
E E\left[\int_{0}^{T}\left(\frac{P_{k}}{l_{t}}\right) d t\right] \leq \int_{0}^{T} E E\left[\left(\frac{P_{k}}{l_{t}}\right)\right] d t,
$$

where $l_{t}$ denotes a time interval length. Furthermore, assume a suboptimal solution with value $\hat{P}_{t}$ existing, which of course, a constant value with $\hat{P}_{t}>0, t \in(t(i-$ $1), t(i)]$ existing. We have

$$
\begin{aligned}
& \mathrm{EE} \int_{0}^{T}\left[\left(\frac{P_{k}-\hat{P}_{k}}{l_{t}}\right)\right] d t \\
& \geq \int_{0}^{T}\left\{\left[\operatorname{EE}\left(\frac{P_{k}}{l_{t}}\right)-\operatorname{EE}\left(\frac{\hat{P}_{k}}{l_{t}}\right)\right]\right\} d t .
\end{aligned}
$$

However, according to the optimization results, there should be

$$
\begin{aligned}
& \operatorname{EE} \int_{0}^{T}\left[\left(\frac{P_{k}-\hat{P}_{k}}{l_{t}}\right)\right] d t \\
& \leq \int_{0}^{T}\left[\operatorname{EE}\left(\frac{P_{k}}{l_{t}}\right)-\operatorname{EE}\left(\frac{\hat{P}_{k}}{l_{t}}\right)\right] d t .
\end{aligned}
$$


As we can see, this resulted in a paradox. Thus, $\hat{P}_{k}$ must be equal to $P_{k}$ in order to get one and only one integral optimal result. In this case, the global optimal result of EE can be obtained by the sum optimal power in each independent time period. This completes the proof.

\section{Acknowledgements}

This work is supported by the EU-JAPAN co-funded project 5G Pagoda (European Commission H2020 program under grant agreement no. 723172); research and development on fundamental and utilization technologies for social big data, NICT, Japan; research on big data dynamic parallel cooperative processing function using autonomous distributed M2M network (MIC Scope Type II, Phase I, 2015).

This paper is based on the paper accepted by IEEE ICC 2016 with title "Integrating Energy Efficiency Mechanism with Components Selection for Massive MIMO Based C-RAN."

\section{Competing interests}

The authors declare that they have no competing interests.

\section{Author details}

${ }^{1}$ GITS/GITI, Waseda University, Okubo, 3-14-8, 169-0074 Tokyo, Japan.

${ }^{2}$ Department of Electrical Engineering, Princeton University, Princeton 08540,

NJ, USA. ${ }^{3}$ Instituto de Telecomunicaçōes, 3810-193 Aveiro, Portugal.

Received: 22 April 2016 Accepted: 22 November 2016

Published online: 03 December 2016

\section{References}

1. Q Wu, M Tao, WK Ng, W Chen, R Schober, Energy-efficient resource allocation for wireless powered communication networks. IEEE Trans. Wireless Commun. 15(3), 2312-2327 (2016)

2. D Zhang, S Mumtaz, Z Zhou, T Sato, in IEEE ICC. Integrating energy efficiency mechanism with components selection for massive MIMO based C-RAN, (Kuara Lumpur, Malaysia, 2016)

3. J Gong, S Zhou, Z Niu, Optimal power allocation for energy harvesting and power grid coexisting wireless communication systems. EEE Trans. Commun. 61(7), 3040-3049 (2013)

4. Z Chang, J Gong, Y Li, Z Zhou, T Ristaniemi, G Shi, Z Han, Z Niu, Energy Efficient Resource Allocation for Wireless Power Transfer Enabled Collaborative Mobile Clouds. IEEE Journal on Selected Areas in Communications. PP(99) (2016)

5. Z Niu, Y Wu, J Gong, Z Yang, Cell zooming for cost efficient green cellular networks. IEEE Commun. Mag. 48(11), 74-79 (2010)

6. L Thiele, T Wirth, M Schellmann, Y Hadisusanto, V Jungnickel, in IEEE ICC Workshops. Mu-mimo with localized downlink base station cooperation and downtilted antennas, (2009), pp. 1-5

7. Z Zhou, M Dong, K Ota, CXu, Energy-efficient matching for resource allocation in D2D enabled cellular networks. IEEE Trans. Veh. Tech. PP(99), 1-12 (2016)

8. S ZHANG, Y WU, S Zhou, Z Niu, Traffic-aware network planning and green operation with bs sleeping and cell zooming. IEICE Trans. Commun. E97-B(11), 2118-2126 (2014)

9. X Zhang, S Zhou, Z Niu, X Lin, in IEEE WCNC. An energy-efficient user scheduling scheme for multiuser MIMO systems with RF chain sleeping, (2013), pp. 169-174

10. M Ju, HK Song, IM Kim, Joint relay-and-antenna selection in multi-antenna relay networks. IEEE Trans. Commun. 58(12), 3417-3422 (2010)

11. D Zhang, KYu, ZZhou, T Sato, in IEEE ISADS. Energy efficiency scheme with cellular partition zooming for massive MIMO systems, (2015), pp. 266-271

12. E Bjornson, L Sanguinetti, J Hoydis, M Debbah, Optimal design of energy-efficient multi-user mimo systems: is massive MIMO the answer? IEEE Trans.Wireless Commun. 14(6), 3059-3075 (2015)

13. China Mobile, C-RAN, The Road Towards Green RAN. (2014). http://labs. chinamobile.com/cran/wp-content/uploads/CRAN_white_paper_v2_5_ EN.pdf

14. I Alyafawi, E Schiller, T Braun, D Dimitrova, A Gomes, N Nikaein, in IEEE ICC. Critical issues of centralized and cloudified LTE-FDD radio access networks, (2015), pp. 5523-5528
15. KM Saidul Huq, S Mumtaz, J Bachmatiuk, J Rodriguez, X Wang, RL Aguiar, Green HetNet comp: energy efficiency analysis and optimization. IEEE Trans. Veh. Technol. 64(10), 4670-4683 (2015)

16. S Zhou, T Zhao, Z Niu, Software-defined hyper-cellular architecture for green and elastic wireless access. IEEE Commun. Mag. 54(1), 12-19 (2016)

17. J Xu, L Duan, R Zhang, Cost-aware green cellular networks with energy and communication cooperation. IEEE Commun. Mag. 53(5), 257-263 (2015)

18. M Jung, T Kim, K Min, Y Kim, J Lee, S Choi, in IEEE VTC. Asymptotic distribution of system capacity in multiuser MIMO systems with large number of antennas, (2013), pp. 1-5

19. F Rusek, D Persson, BK Lau, EG Larsson, TL Marzetta, O Edfors, F Tufvesson, Scaling up MIMO: opportunities and challenges with very large arrays. IEEE Signal Processing Mag. 30(1), 40-60 (2013)

20. TL Narasimhan, A Chockalingam, Channel hardening-exploiting message passing (CHEMP) receiver in large-scale MIMO systems. IEEE J. Sel. Topics Signal Process. 8(5), 847-860 (2014)

21. HChen, Y Jiang, J Xu, H Hu, Energy-efficient coordinated scheduling mechanism for cellular communication systems with multiple component carriers. IEEE J. Sel. Areas Commun. 31(5), 959-968 (2013)

22. J Xu, R Zhang, Throughput optimal policies for energy harvesting wireless transmitters with non-ideal circuit power. IEEE J. Sel. Areas Commun. 32(2), 322-332 (2014)

23. Z Zhou, S Zhou, J Gong, Z Niu, in IEEE GLOBECOM. Energy-efficient antenna selection and power allocation for large-scale multiple antenna systems with hybrid energy supply, (2014), pp. 2574-2579

24. $X$ Chen, $X$ Wang, $X$ Chen, Energy-efficient optimization for wireless information and power transfer in large-scale MIMO systems employing energy beamforming. IEEE Wireless Commun. Lett. 2(6), 667-670 (2013)

25. OLM MC Ferris, SJ Wright. Linear Programming with MATLAB (SIAM, Philadelphia, 2007)

26. D Zhu, M Lei, in CHINACOM. Traffic adaptation and energy saving potential of centralized radio access networks with coordinated resource allocation and consolidation, (2013), pp. 587-593

\section{Submit your manuscript to a SpringerOpen ${ }^{\circ}$ journal and benefit from:}

- Convenient online submission

- Rigorous peer review

- Immediate publication on acceptance

- Open access: articles freely available online

- High visibility within the field

- Retaining the copyright to your article

Submit your next manuscript at springeropen.com 